\title{
Estimating zero-sequence impedance of three-terminal transmission line and Thevenin impedance using relay measurement data
}

\author{
Swagata Das ${ }^{1}$, Sundaravaradan Navalpakkam Ananthan ${ }^{2 *}$ (D) and Surya Santoso ${ }^{2}$
}

\begin{abstract}
Current and voltage waveforms recorded by intelligent electronic devices (IEDs) are more useful than just performing post-fault analysis. The objective of this paper is to present techniques to estimate the zero-sequence line impedance of all sections of a three-terminal line and the Thevenin equivalent impedance of the transmission network upstream from the monitoring location using protective relay data collected during short-circuit ground fault events. Protective relaying data from all three terminals may not be always available. Furthermore, the data from each terminal may be unsynchronized and collected at different sampling rates with dissimilar fault time instants. Hence, this paper presents approaches which use unsynchronized measurement data from all the terminals as well as data from only two terminals to estimate the zero-sequence line impedance of all the sections of a three-terminal line. An algorithm to calculate positive-, negative- and zero-sequence Thevenin impedance of the upstream transmission network has also been presented in this paper. The efficacy of the proposed algorithms are demonstrated using a test case. The magnitude error percentage in determining the zero-sequence impedance was less than $1 \%$ in the test case presented.
\end{abstract}

Keywords: Zero-sequence, Three-terminal line, Thevenin Impedance

\section{Introduction}

One of the key features in modern intelligent electronic devices (IEDs) such as digital relays and digital fault recorders is the generation of event reports during faults. By analyzing fault event reports, system operators can understand what happened during the event and the cause of the event but event reports contains much more valuable information. The authors in [1-3] have used event reports to glean information about relay misoperations and estimate a variety of system parameters.

Transmission line parameter estimation using different methods and a variety of data has been a topic of interest for researchers $[4,5]$. The zero-sequence impedance of an overhead line must be specified by protection engineers in relay settings and plays a key role in distance and directional protection $[6,7]$, and fault location calculations.

\footnotetext{
*Correspondence: varadan@utexas.edu

${ }^{2}$ Department of Electrical and Computer Engineering, The University of Texas, Austin, USA

Full list of author information is available at the end of the article
}

The zero-sequence impedance of a line depends on earth resistivity. A commonly used practice to determine the zero-sequence impedance is by using Carson's equation and using a typical value of $100 \Omega$-m as earth resistivity $[6,8]$. As the earth resistivity depends on various factors such as soil type, temperature and moisture content in the soil [9] and is difficult to measure accurately, the zerosequence line impedance is subject to much uncertainty. As a result, to avoid relay mis-operations and incorrect fault location, efforts must be made to validate the zerosequence line impedance using fault event data.

Three-terminal transmission lines are frequently used by utilities to transfer bulk electrical power and support loads from three generating sources [10]. Often, utilities upgrade an existing two-terminal line to a three-terminal line by simply connecting a line with a generating source to it. Building three-terminal lines have several advantages. There are low or no costs associated with constructing a new substation and procuring power system equipment. No right-of-way and regulatory approvals are required. As a result, three-terminal lines are expeditious 
in increasing the operational support necessary to meet system demands.

Several authors in the past have researched on validating zero-sequence line impedance but only for two-terminal lines $[6,11]$. Very little work, if any, has been conducted on validating the zero-sequence line impedance of threeterminal transmission lines. The event reports from IEDs at different ends of the line may detect the fault at slightly different time instants and have different sampling rates. Furthermore, they can be unsynchronized. Therefore, it is necessary to devise a methodology that can use unsynchronized measurements to confirm the zero-sequence impedance of three-terminal transmission lines.

Using the same set of waveform data, the Thevenin impedance of the transmission network upstream from the monitoring location can be estimated. The Thevenin impedance, often referred to as the short-circuit impedance, plays an important role when calculating the currents during a balanced or an unbalanced fault [12]. System operators obtain the Thevenin impedance by performing a short-circuit analysis on the circuit model. However, to avoid any erroneous fault current calculations due to an inaccurate circuit model, it is a good practice to validate the short-circuit impedance from the circuit model with that calculated from the fault data. The Thevenin impedance is also required by the Eriksson, Novosel et al., and other fault-locating algorithms to track down the location of a fault [8, 13, 14]. Furthermore, the Thevenin impedance calculated at regular intervals during a long duration fault can provide insight into the state of the transmission network upstream from the fault. Authors in [15] have attempted to estimate the Thevenin impedance but have not provided the equations for the same. Authors in $[15,16]$ have also highlighted the importance and uses of estimating the Thevenin impedance. Hence, it is essential to estimate the Thevenin impedance of the transmission network upstream from the monitoring location using any available fault data.

Based on the aforementioned background, the primary objective of this paper is to estimate the zero-sequence impedance of three-terminal line from waveform data captured by intelligent electronic devices during a ground fault. The contribution lies in developing algorithms which either use unsynchronized data from all the three terminals or use unsynchronized data from only two terminals. The second objective of this paper is to estimate the Thevenin impedance of the transmission network upstream from the monitoring location using the same set of fault waveform data. The authors had developed algorithms for estimating zero-sequence line impedance for two-terminal lines [5] but this paper explores algorithms for estimating zero-sequence impedance of threeterminal lines which has barely been studied previously.
This paper serves as a further extension and as a sequel to the aforementioned paper.

The proposed methods for both estimating the zerosequence line impedance as well as estimating Thevenin impedance from fault waveforms are validated using a test case. The approach which estimates zero-sequence line impedance using data from three terminals requires lesser assumptions than the method which uses data from only two terminals. Both the methods produced accurate zerosequence impedance estimates and the magnitude error percentage was less than $1 \%$. Similarly, results indicate the success of Thevenin impedance estimation algorithm as the magnitude error was less than $1 \%$ in the test case.

\section{Estimating the zero-sequence impedance of three-terminal lines}

Three-terminal lines do pose a significant challenge to the task of validating the zero-sequence line impedance. The third terminal contributes to the total fault current and changes the impedance equations which are commonly used for two-terminal lines. Furthermore, with the introduction of a third terminal, there are now two lines whose zero-sequence line impedances have to be validated from a single fault event. Based on this aforementioned background, this section presents two approaches for calculating the zero-sequence line impedance of threeterminal lines. The proposed algorithm uses fault current and voltage phasors from the line terminals for its analysis. The proposed technique should therefore be applied on the steady state portion of fault waveforms to obtain accurate results. Hence, it is suitable for application in ground fault scenarios which contain steady state fault waveforms. Approach 1 requires the availability of voltage and current waveforms from all the three terminals while Approach 2 uses waveforms captured at two terminals only.

\subsection{Approach 1 for estimating zero-sequence line impedance: data from three terminals}

This approach requires the availability of voltage and current phasors at all the three terminals during a fault and is illustrated using the three-terminal transmission line shown in Fig. 1. Line 2 is homogeneous and connects terminal $\mathrm{G}$ with terminal $\mathrm{H}$. Line 2 is of length LL per unit. Line 1 is also homogeneous and connects terminal $\mathrm{T}$ with Line 2 at a distance of $d$ per unit from terminal G. When a single or double line-to-ground fault occurs on Line 2 at $m$ per unit distance from terminal $\mathrm{G}$, all three sources contribute to the fault. Digital relays at each terminal capture the voltage and current phasors during the fault. The phasors need not be synchronized with each other.

The steps to validate the zero-sequence impedance of Line 1 and Line 2 are outlined below. 


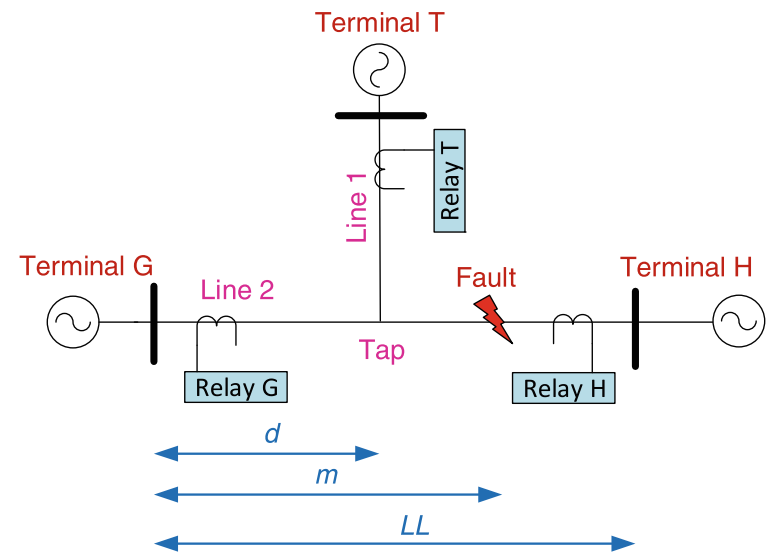

Fig. 1 Three-terminal transmission line model used for analysis

\subsubsection{Step 1: synchronize terminal $T$ with terminal $G$}

Consider the negative-sequence network of a threeterminal line during a single or a double line-to-ground fault as shown in Fig. 2. Let $\delta 1$ represent the synchronization error between the measurements at terminal $\mathrm{T}$ and terminal G. Therefore, to align the voltage and current phasors at terminal $\mathrm{T}$ with respect to terminal $\mathrm{G}$, a synchronization operator, $e^{j \delta 1}$, is applied to the terminal $\mathrm{T}$ measurements. The value of $e^{j \delta 1}$ can be determined by calculating $V_{\text {Tap2 }}$ from both terminals as

$$
\begin{aligned}
& \text { Terminal G: }: V_{\text {Tap } 2}=V_{G 2}-\left(d \times Z_{2} L_{2} \times I_{G 2}\right) \\
& \text { Terminal } T: V_{T a p 2}=V_{T 2} e^{j \delta 1}-\left(Z_{2} L_{1} \times I_{T 2} e^{j \delta 1}\right)
\end{aligned}
$$

Notations in the Figs. 2 and 3 are defined as follows:

$V_{\text {Tap2 }}$

$V_{G 2}, V_{H 2}, V_{T 2}$

$I_{G 2}, I_{H 2}, I_{T 2}$

$V_{G 0}, V_{H 0}, V_{T 0}$

$I_{G 0}, I_{H 0}, I_{T 0}$ is the negative-sequence voltage at the tap point are the negative-sequence fault voltages at terminals $\mathrm{G}, \mathrm{H}$ and $\mathrm{T}$ respectively are the negative-sequence fault currents at terminals $\mathrm{G}, \mathrm{H}$ and $\mathrm{T}$ respectively are the zero-sequence fault voltages at terminals $\mathrm{G}, \mathrm{H}$ and $\mathrm{T}$ respectively are the zero-sequence fault currents at terminals $\mathrm{G}, \mathrm{H}$ and $\mathrm{T}$ respectively

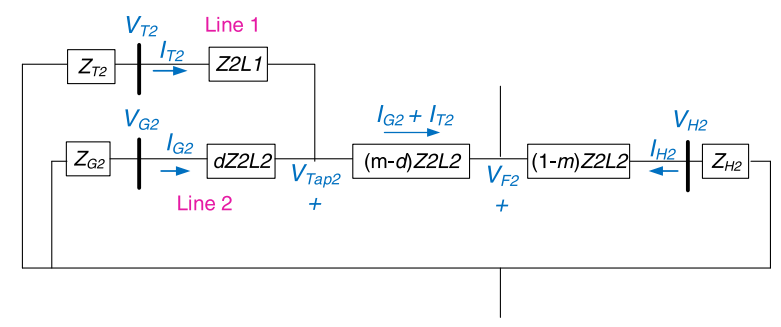

Fig. 2 Negative-sequence network of the three-terminal line in Fig. 1 during a single or double line-to-ground fault

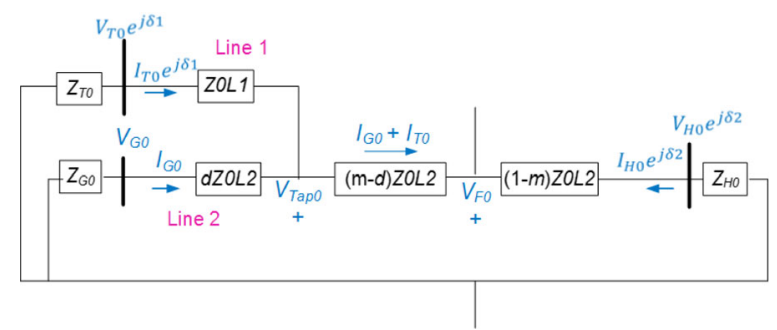

Fig. 3 Zero-sequence network of the three-terminal line in Fig. 1 during a single or double line-to-ground fault

$\begin{array}{ll}Z_{2} L_{1} & \text { is the negative-sequence impedance of } \\ & \text { Line } 1 \\ Z_{2} L_{2} & \text { is the negative-sequence impedance of } \\ & \text { Line } 2 \\ Z_{0} L_{1} & \text { is the zero-sequence impedance of Line } 1 \\ Z_{0} L_{2} & \text { is the zero-sequence impedance of Line } 2\end{array}$

Since terminals $G$ and $T$ operate in parallel to feed the fault, $V_{\text {Tap } 2}$ should be equal when calculated from either terminal. From this principle, $e^{j \delta 1}$ can be solved as

$$
e^{j \delta 1}=\frac{V_{G 2}-\left(d \times Z_{2} L_{2} \times I_{G 2}\right)}{V_{T 2}-\left(Z_{2} L_{1} \times I_{T 2}\right)}
$$

In effect, this step calculates the phase angle mismatch between terminal $\mathrm{G}$ and terminal $\mathrm{T}$ measurements, and accordingly adjusts the phasors at terminal $\mathrm{T}$ by $e^{j \delta 1}$.

2.1.2 Step 2: synchronize terminal $H$ with terminals $T$ and $G$ After the synchronization process in Step 1, the new negative-sequence voltage and current phasors at terminal $\mathrm{T}$ are $V_{T 2} e^{j \delta 1}$ and $I_{T 2} e^{j \delta 1}$, respectively, and at terminal $\mathrm{G}$ are $V_{G 2}$ and $I_{G 2}$, respectively. This step synchronizes the phasors at terminal $\mathrm{H}$ with the phasors at terminals $\mathrm{G}$ and T. For this purpose, a second synchronizing operator, $e^{j \delta 2}$, is applied to the measurements at terminal $\mathrm{H}$. The value of $e^{j \delta 2}$ can be calculated from the fact that the negative-sequence voltage at the fault point, $V_{F 2}$, must be the same when calculated from either terminal $\mathrm{G}$ or $\mathrm{H}$ as

TerminalG :

$V_{F 2}=V_{G 2}-\left[m Z_{2} L_{2} \times I_{G 2}\right]-\left[(m-d) Z_{2} L_{2} \times I_{T 2} e^{j \delta 1}\right]$

TerminalH :

$V_{F 2}=V_{H 2} e^{j \delta 2}-\left[(1-m) Z_{2} L_{2} \times I_{H 2} e^{j \delta 2}\right]$

Therefore, $e^{j \delta 2}$ is given by

$$
e^{j \delta 2}=\frac{V_{G 2}-\left[m Z_{2} L_{2} \times I_{G 2}\right]-\left[(m-d) Z_{2} L_{2} \times I_{T 2} e^{j \delta 1}\right]}{V_{H 2}-\left[(1-m) Z_{2} L_{2} \times I_{H 2}\right]}
$$


At the end of this step, the voltage and current measurements at terminals $\mathrm{T}$ and $\mathrm{H}$ are synchronized with respect to those at terminal G.

\subsubsection{Step 3: calculate the zero-sequence impedance of Line 2}

To estimate the zero-sequence impedance of Line 2, the zero-sequence network during a single or double line-toground fault is shown in Fig. 3. The zero-sequence voltage at the fault point, $V_{F 0}$, can be calculated from terminal $\mathrm{G}$ and $\mathrm{H}$ as

\section{TerminalG :}

$V_{F 0}=V_{G 0}-\left(m Z_{0} L_{2} \times I_{G 0}\right)-\left[(m-d) Z_{0} L_{2} \times I_{T 0} e^{j \delta 1}\right]$

TerminalH :

$$
V_{F 0}=V_{H 0} e^{j \delta 2}-\left[(1-m) Z_{0} L_{2} \times I_{H 0} e^{j \delta 2}\right]
$$

Since $V_{F 0}$ is equal when calculated from either line terminal, equate the two equations in (5) to solve for $Z_{0} L_{2}$ as

$$
Z_{0} L_{2}=\frac{V_{G 0}-V_{H 0} e^{j \delta 2}}{m I_{G 0}+(m-d) I_{T 0} e^{j \delta 1}-(1-m) I_{H 0} e^{j \delta 2}}
$$

\subsubsection{Step 4: calculate the zero-sequence impedance of Line 1}

To estimate the zero-sequence impedance of Line $1, Z_{0} L_{1}$, calculate the zero-sequence voltage at the tap point, $V_{T a p 0}$, from terminals $\mathrm{G}$ and $\mathrm{T}$ as

$$
\begin{aligned}
& \text { Terminal G: }: V_{T a p 0}=V_{G 0}-\left(d Z_{0} L_{2} \times I_{G 0}\right) \\
& \text { Terminal } T: V_{\text {Tap } 0}=V_{T 0} e^{j \delta 1}-\left(Z_{0} L_{1} \times I_{T 0} e^{j \delta 1}\right)
\end{aligned}
$$

where $V_{T 0}$ is the zero-sequence fault voltages at terminal T. Because $V_{\text {Tap } 0}$ from terminal $\mathrm{G}$ is equal to that from terminal $\mathrm{T}$, we can use (7) to solve for $Z_{0} L_{1}$ as

$$
Z_{0} L_{1}=\frac{V_{T 0} e^{j \delta 1}-V_{G 0}+\left(d Z_{0} L_{2} \times I_{G 0}\right)}{I_{T 0} e^{j \delta 1}}
$$

In this way, Approach 1 is successful in validating the zero-sequence impedance of Line 1 and Line 2 . If the actual fault location is not available, then an additional step (Step 0) must be performed to track down the exact fault location before applying the steps described above.

\subsubsection{Step 0: identify the fault location}

Before computing the distance to the fault, it is necessary to identify whether the fault is on Line 1 or on Line 2 . The negative-sequence network shown in Fig. 2 is used for this purpose. When the fault is between terminal $\mathrm{H}$ and the tap point, $V_{T a p 2}$ from the other two terminals are equal. Therefore, to identify the faulted section of the feeder, the approach consists of calculating $V_{\text {Tap2 }}$ from each terminal as

$$
\begin{aligned}
& \text { Terminal G: }\left|V_{\text {Tap } 2}\right|=\left|V_{G 2}-\left(d \times Z_{2} L_{2} \times I_{G 2}\right)\right| \\
& \text { Terminal } H:\left|V_{\text {Tap } 2}\right|=\left|V_{H 2}-\left((L L-d) \times Z_{2} L_{2} \times I_{H 2}\right)\right| \\
& \text { Terminal } T:\left|V_{\text {Tap } 2}\right|=\left|V_{T 2}-\left(Z_{2} L_{1} \times I_{T 2}\right)\right|
\end{aligned}
$$

The estimated $\left|V_{\text {Tap2 } 2}\right|$ from two of the terminals will be an exact match while $\left|V_{\operatorname{Tap} 2}\right|$ from the third terminal will be different. The fault is expected to lie between that third terminal and the tap point. Next, apply one-ended fault location algorithm to the voltage and current waveforms at the third terminal and estimate the distance to the fault.

Figure 4 shows a flow diagram which summarizes Approach 1 for calculating the zero-sequence line impedance.

\subsection{Assumptions}

Assumptions made by Approach 1 when estimating the zero-sequence impedances of Line 1 and Line 2 are summarized below:

1. Line 1 and Line 2 are homogeneous

2. Zero-sequence mutual coupling is absent

A transmission network is homogeneous when the local and remote source impedance have the same impedance angle as the transmission line. Assumption 1 is a reasonable assumption to make as local and remote source impedance angles are often close to that of the transmission line.

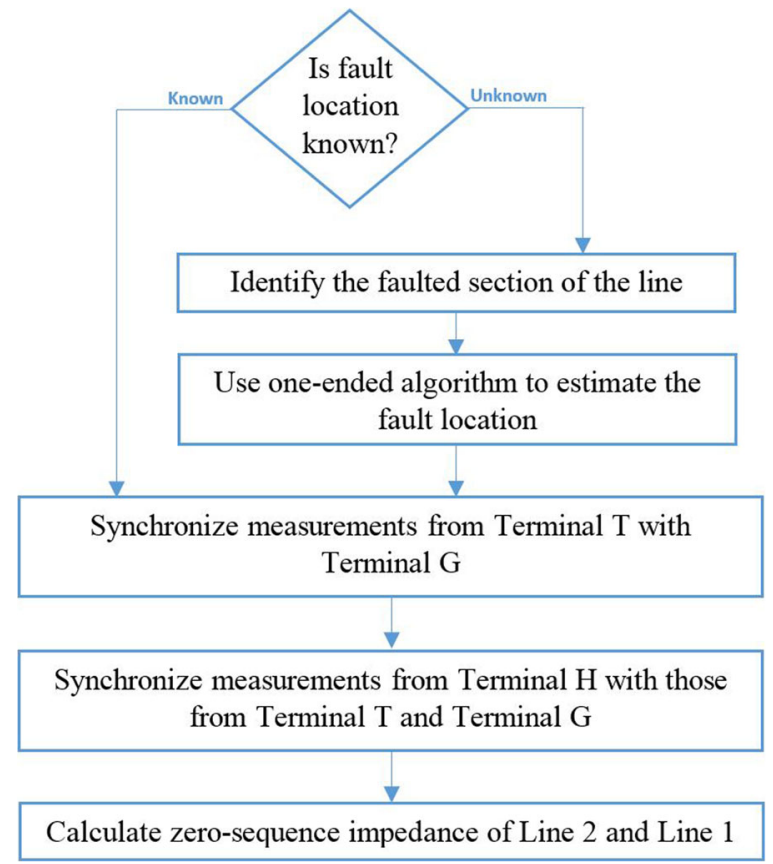

Fig. 4 Flow diagram depicting Approach 1 for calculating zero-sequence line impedance of all the sections of a three-terminal line using data from all three terminals 
Zero-sequence mutual coupling arises when transmission lines are physically very close or parallel to each other. An example where zero-sequence mutual coupling could be present is when there are two sets of three-phase lines running on same towers. Though this configuration of lines is occasionally found in two-terminal transmission lines, it is highly unlikely and rare to have three-terminal transmission line with such a wire configuration that would have zero-sequence mutual coupling.

\subsection{Approach 2 for estimating zero-sequence line impedance: data from two terminals}

Although Approach 1 can successfully validate the zerosequence impedance of three-terminal transmission lines, voltage and current waveforms from all the three terminals may not always be available. For this reason, this subsection develops a methodology that can use data from only two terminals to estimate the zerosequence impedance of three-terminal lines. To illustrate the approach, consider the scenario shown in Fig. 1. Suppose that the measurements captured by digital relays at terminals $\mathrm{G}$ and $\mathrm{H}$ are available while measurements from terminal $\mathrm{T}$ are missing. The procedure to estimate the zero-sequence impedance of Line 1 and Line 2 in such a scenario is described below:

\subsubsection{Step 1: estimate the negative-sequence current from terminal $T$}

To estimate the negative-sequence fault current from terminal $\mathrm{T}, I_{T 2}$, the negative-sequence network shown in Fig. 2 is used. The approach consists of calculating $V_{\text {Tap } 2}$ from terminal $\mathrm{G}$ and terminal $\mathrm{T}$ as

$$
\begin{aligned}
& \text { Terminal G: } V_{\text {Tap } 2}=V_{G 2}-\left(d \times Z_{2} L_{2} \times I_{G 2}\right) \\
& \text { Terminal } T: V_{\text {Tap } 2}=-\left(Z_{T 2}+Z_{2} L_{1}\right) I_{T 2}
\end{aligned}
$$

where $Z_{T 2}$ is the negative-sequence source impedance of terminal T. Since $V_{\text {Tap } 2}$ is equal when calculated from either terminal, $I_{T 2}$ can be estimated as

$$
I_{T 2}=\frac{\left(d \times Z_{2} L_{2} \times I_{G 2}\right)-V_{G 2}}{Z_{T 2}+Z_{2} L_{1}}
$$

Note that since terminal $G$ measurements are being used to estimate the fault current from terminal $\mathrm{T}$, measurements of these two terminals are automatically synchronized.

\subsubsection{Step 2: synchronize terminal $H$ with terminals $G$ and $T$} This step applies a synchronization operator, $e^{j \delta}$, to the terminal $\mathrm{H}$ measurements so as to align the measure- ments at this terminal with those at terminals $\mathrm{G}$ and $\mathrm{T}$. The fact that $V_{F 2}$ is the same when calculated from terminals $\mathrm{G}$ and $\mathrm{H}$ is used to calculate the synchronization operator as

$$
e^{j \delta}=\frac{V_{G 2}-\left(m Z_{2} L_{2} \times I_{G 2}\right)-\left[(m-d) \times Z_{2} L_{2} \times I_{T 2}\right]}{V_{H 2}-\left[(1-m) Z_{2} L_{2} \times I_{H 2}\right]}
$$

The new set of sequence voltage phasors at terminal $\mathrm{H}$ are $V_{H 1} e^{j \delta}, V_{H 2} e^{j \delta}$, and $V_{H 0} e^{j \delta}$. Similarly, the new set of current phasors at terminal $\mathrm{H}$ are $I_{H 1} e^{j \delta}, I_{H 2} e^{j \delta}$, and $I_{H 0} e^{j \delta}$.

\subsubsection{Step 3: estimate the zero-sequence impedance of line 2}

The zero-sequence line impedance of Line 2 is estimated from terminal $\mathrm{H}$ measurements using any zero-sequence line impedance estimation algorithm for two-terminal line which uses measurements from one terminal only [5, 11]. The algorithm to estimate the zero-sequence line impedance of a two-terminal line using data from only one terminal assumes that the fault resistance is zero and the fault location is known.

\subsubsection{Step 4: estimate the zero-sequence current from terminal $T$}

The zero-sequence voltage at the fault point, $V_{F 0}$, is the same when calculated from terminal $G$ or terminal $H$. This principle is used to calculate the zero-sequence fault current contributed by terminal $\mathrm{T}, I_{T 0}$, as

$$
I_{T 0}=\frac{V_{G 0}-V_{H 0} e^{j \delta}+Z_{0} L_{2}\left[(1-m) I_{H 0} e^{j \delta}-m I_{G 0}\right]}{Z_{0} L_{2}(m-d)}
$$

2.3.5 Step 5: estimate the zero-sequence impedance of line 1 The fact that $V_{\text {Tap } 0}$ is the same when calculated from terminal $\mathrm{G}$ or terminal $\mathrm{T}$ is used to estimate the zerosequence impedance of Line 1 as

$$
Z_{0} L_{1}=\frac{\left(d \times Z_{0} L_{2} \times I_{G 0}\right)-V_{G 0}}{I_{T 0}}-Z_{T 0}
$$

Figure 5 shows a flow diagram which summarizes Approach 2 for calculating the zero-sequence line impedance.

\subsection{Assumptions:}

Assumptions made by Approach 2 when estimating the zero-sequence line impedance are summarized below:

1. Line 1 and Line 2 are homogeneous

2. Fault location is known

3. Fault resistance is zero

4. Zero-sequence mutual coupling is absent

The assumptions that the transmission network is homogeneous and the absence of zero-sequence mutual coupling are same as that of Approach 1. Since Approach 
Calculate negative-sequence current from Terminal T

Synchronize measurements from Terminal $\mathrm{H}$ with those from Terminal $\mathrm{T}$ and Terminal $\mathrm{G}$

Estimate zero-sequence impedance of Line 2 using any zero-sequence line impedance estimation algorithm for two-terminal line which uses measurements from one terminal only

Estimate zero-sequence current from Terminal T

Estimate zero-sequence impedance of Line 1

Fig. 5 Flow diagram depicting Approach 2 for calculating zero-sequence line impedance of all the sections of a three-terminal line using data from only two terminals

2 has lesser data availability than Approach 1, further assumptions must be made to obtain the zero-sequence line impedance of the lines.

In utility/industry practice, the process of estimating the zero-sequence line impedance is an offline post fault analysis process using event reports recorded by the relay. The electric power utility performs a thorough investigation of the fault event as soon as the fault has occurred. As a result, it is expected that the utility identifies the fault location and hence, it is a reasonable assumption that the fault location is known while implementing the proposed algorithm.

It is highly unlikely to have a completely bolted fault where the fault resistance is exactly zero. However, it is a reasonable assumption to make when the fault resistance value is small and close to zero. Though not every short-circuit fault has a fault impedance value close to zero, the occurrence of this type of fault where the fault impedance is close to zero is relatively common in transmission lines. Such a scenario has also been demonstrated and analyzed using real-world field data in [5]. However, having to extract more information from less data requires us to make such an assumption.

\section{Demonstration of estimation of zero-sequence impedance using a test case}

This section demonstrates the efficacy of Approach 1 and Approach 2 in estimating the zero-sequence line impedance of a three-terminal line during a single line-toground fault. The system parameters used for the study are described below.

The three-terminal network shown in Fig. 1 was modeled in PSCAD simulation software [17]. The model was used to replicate actual short-circuit faults that occur on a transmission line and generate the corresponding voltage and current waveforms.

The positive- and zero-sequence source impedances at terminal $G$ are $Z_{G 1}=3.75 \angle 86^{\circ} \Omega$ and $Z_{G 0}=11.25 \angle 86^{\circ} \Omega$, respectively. The positive- and zero-sequence source impedances at terminal $\mathrm{H}$ are $Z_{H 1}=12 \angle 80^{\circ} \Omega$ and $Z_{H 0}=36 \angle 80^{\circ} \Omega$, respectively. The positive- and zerosequence source impedances at terminal $\mathrm{T}$ are $Z_{T 1}=$ $5 \angle 83^{\circ} \Omega$ and $Z_{T 0}=12 \angle 83^{\circ} \Omega$, respectively.

The distance between terminal $G$ and the tap point is 6.21 miles. Line 2 is 18.64 miles long and has the same configuration as shown in Fig. 6. Shield wires $\mathrm{S}_{1}$ and $\mathrm{S}_{2}$ protect phase conductors $\mathrm{A}, \mathrm{B}$, and $\mathrm{C}$ from direct lightning strikes. The positive- and zerosequence line impedances calculated using modified Carson's model [18] at an earth resistivity value of $100 \Omega$ $\mathrm{m}$ are $Z_{1} L_{2}=16.15 \angle 70^{\circ} \Omega$ and $Z_{0} L_{2}=34.87 \angle 64^{\circ} \Omega$, respectively. Line 1 is 6.21 miles long and also has the same configuration as Fig. 6. However, the earth resistivity was changed to $80 \Omega-\mathrm{m}$. The positive- and zerosequence line impedances are $Z_{1} L_{1}=5.38 \angle 70^{\circ} \Omega$ and $Z_{0} L_{1}=11.55 \angle 65^{\circ} \Omega$, respectively.

When a bolted phase A-to-ground fault occurs at a distance of 13 miles from terminal G, monitors at terminals $\mathrm{G}, \mathrm{H}$, and $\mathrm{T}$ capture the voltage and current waveforms at 32,64 , and 128 samples per cycle, respectively. The waveforms are, therefore, not synchronized with each other.

For the test scenarios, the fundamental frequency magnitude and phase angle of the fault current and voltages were extracted using Fast Fourier Transform. To avoid inaccuracies due to transients and DC offset, a cycle with stable fault current is used. For this purpose, the third

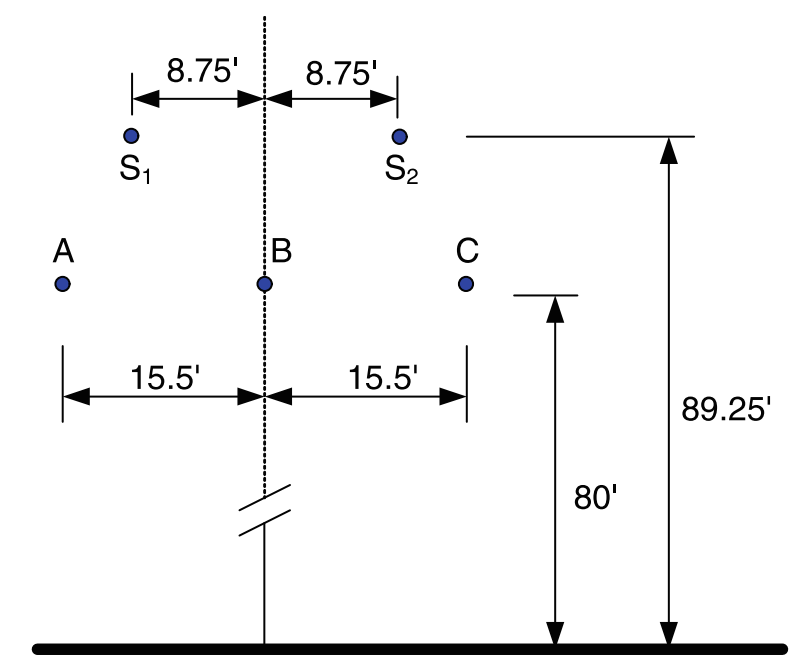

Fig. 6 Tower configuration of an actual 69-kV transmission line model used for demonstrating the proposed algorithms 
cycle after fault inception was chosen in the simulation results to estimate the zero-sequence line impedance. Fast Fourier Transform is a commonly used method to extract fundamental frequency components and filter out decaying DC offset $[8,19]$. If the DC offset remains significant after the third cycle, more advanced filters $[20,21]$ can be used or appropriate fault cycle can be chosen to obtain accurate current and voltage measurements from fault records. This ensures that the error in determining the current and voltage phasors is minimized and hence the zero-sequence line impedance estimation will not be affected by it.

To assess the effectiveness of Approach 1, the fault location is assumed to be unknown. Following Step 0, the negative-sequence voltage magnitude at the tap point, $\left|V_{\text {Tap } 2}\right|$, is calculated to be $4.05 \mathrm{kV}$ from terminal G, $261.78 \mathrm{kV}$ from terminal $\mathrm{H}$, and $4.05 \mathrm{kV}$ from terminal $\mathrm{T}$. Since $\left|V_{\text {Tap } 2}\right|$ from terminals $\mathrm{G}$ and $\mathrm{T}$ are equal, the fault is expected to lie between terminal $\mathrm{H}$ and the tap point. Next, the simple reactance method [8] is applied to the measurements at terminal $\mathrm{H}$ and estimated the distance to the fault to be 13 miles from terminal G or 5.64 miles from terminal $\mathrm{H}$. The next step is to synchronize the measurements at terminals $\mathrm{T}$ and $\mathrm{H}$ with those at terminal $\mathrm{G}$, and estimate the zero-sequence impedances of Line 1 and Line 2. As seen from Table 1, the estimated line impedances match with those used in the simulation test case.

The magnitude and phase angle error were calculated as follows [6]:

$$
\begin{aligned}
\text { Magnitude Error } \% & =\frac{|| \text { Actual } Z_{L 0}|-| \text { Estimated } Z_{L 0}||}{\mid \text { Actual } Z_{L 0} \mid} \times 100 \\
\text { Phase Angle Error } & =\mid \angle \text { Actual } Z_{L 0}-\angle \text { Estimated } Z_{L 0} \mid
\end{aligned}
$$

Approach 2, on the other hand, uses the voltage and current waveforms captured at terminal $\mathrm{G}$ and terminal $\mathrm{H}$, and assumes that the fault location is available. As seen from Table 2, the estimated line impedances are close to those used in the simulation. The magnitude error percentage of the estimated zero-sequence line impedance is less than $1 \%$ for both the approaches.

Although Approach 2 uses data from only two terminals to estimate the zero-sequence line impedance of all the sections of the three-terminal line, it requires the knowledge of source impedance parameters of the

Table 1 Estimated vs. Actual Zero-sequence Line Impedance of Line 1

\begin{tabular}{lllll}
\hline Approach & \multicolumn{2}{l}{$\begin{array}{l}\text { Zero-sequence line } \\
\text { impedance }(\Omega)\end{array}$} & $\begin{array}{l}\text { Magnitude } \\
\text { error (\%) }\end{array}$ & $\begin{array}{l}\text { Phase angle } \\
\text { error (degrees) }\end{array}$ \\
\cline { 2 - 3 } & Actual & Estimated & & \\
\hline Approach 1 & $4.98+j 10.42$ & $4.99+j 10.39$ & 0.19 & 0.11 \\
Approach 2 & & $4.95+j 10.46$ & 0.20 & 0.22 \\
\hline
\end{tabular}

Table 2 Estimated vs. Actual Zero-sequence Line Impedance of Line 2

\begin{tabular}{lllll}
\hline Approach & \multicolumn{2}{l}{$\begin{array}{l}\text { Zero-sequence line } \\
\text { impedance }(\Omega)\end{array}$} & $\begin{array}{l}\text { Magnitude } \\
\text { error (\%) }\end{array}$ & $\begin{array}{l}\text { Phase angle } \\
\text { error (degrees) }\end{array}$ \\
\cline { 2 - 3 } & Actual & Estimated & & \\
\hline Approach 1 & $15.08+j 31.44$ & $15.13+j 31.33$ & 0.22 & 0.15 \\
Approach 2 & & $15.12+j 31.38$ & 0.10 & 0.10 \\
\hline
\end{tabular}

third terminal. For this purpose, the next section provides approaches to calculate the source impedance or the Thevenin impedance of the transmission network upstream from the monitoring location.

In [5], the transmission line, whose zero-sequence estimation was performed, was a two-terminal line whereas three-terminal transmission lines are analyzed in this paper. In a three-terminal transmission line, there is short-circuit current contribution from all three terminals unlike two-terminal transmission line where there is current contribution from only two terminals. Hence, the circuit explored, system equations and method of analysis are different compared to the algorithms presented in [5]. The data availability for a three-terminal line is also different from that of a two-terminal line. Moreover, there is a need to synchronize measurements from different terminals of the line based on data availability before estimating the zero-sequence line impedance. Furthermore, careful system analysis is required to calculate the zero-sequence impedance of both Line 1 and Line 2 as seen in the two approaches discussed above unlike having to estimate the zero-sequence impedance of only one line as in [5]. In addition to estimating the zero-sequence impedance of the lines, this paper also presents a method for estimating the Thevenin impedance of the transmission network upstream from the monition location using the same set of waveform data.

\section{Estimating the Thevenin impedance of upstream network}

Voltage and current waveforms captured by IEDs during a fault can be used to estimate the Thevenin impedance of the transmission network upstream from the monitoring location as illustrated in Fig. 7.

Figures 8, 9 and 10 represent the negative-, zero- and positive-sequence network respectively. The rest of the down stream network is represented as RDSN in Figs. 8, 9 and 10 .

The negative-sequence Thevenin impedance, $Z_{G 2}$, can be calculated from the negative-sequence network shown in Fig. 8 during an unbalanced fault as

$$
Z_{G 2}=-\frac{V_{G 2}}{I_{G 2}}
$$




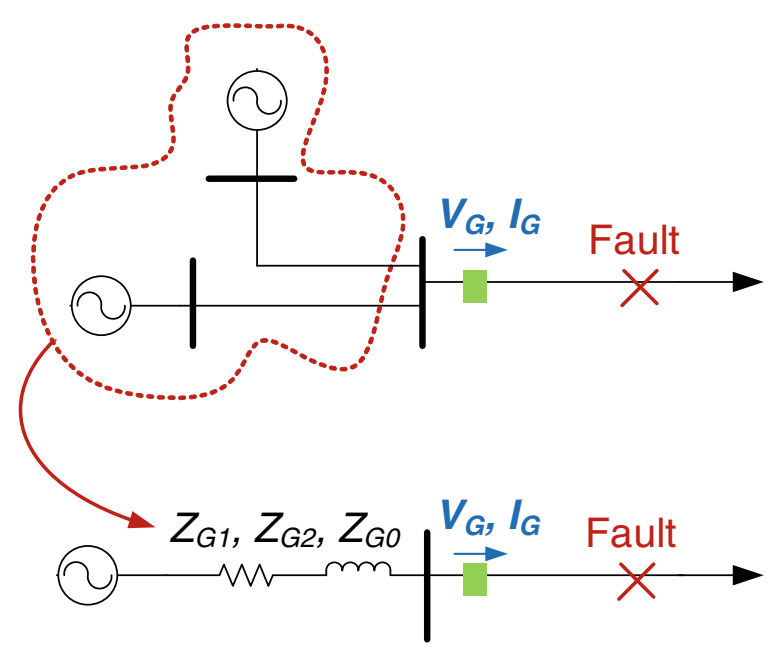

Fig. 7 Concept of Thevenin impedance. The effect of the impedance of the upstream network can be emulated by replacing it with an equivalent impedance and this equivalent impedance is called Thevenin impedance

In a similar manner, the zero-sequence source impedance, $Z_{G 0}$, can be calculated from the zero-sequence network in Fig. 9 during a ground fault as

$$
Z_{G 0}=-\frac{V_{G 0}}{I_{G 0}}
$$

The calculation of the positive-sequence source impedance, $Z_{G 1}$, on the other hand, is complicated by the presence of an internal generator voltage, $E_{G}$, as shown in Fig. 10. As a workaround, the superposition principle is used to decompose the network into a prefault and "pure fault" network. Impedance $Z_{G 1}$ can be estimated from the "pure fault" network as

$$
Z_{G 1}=-\frac{\Delta V_{G}}{\Delta I_{G}}=-\frac{V_{G 1}-V_{G 1 p r e}}{I_{G 1}-I_{G 1 p r e}}
$$

It should be noted that $Z_{G 1}$ is not assumed to be equal to $Z_{G 2}$ and is calculated separately using (19). This is because the positive-sequence impedance equals the negative-sequence impedance in static electrical com-

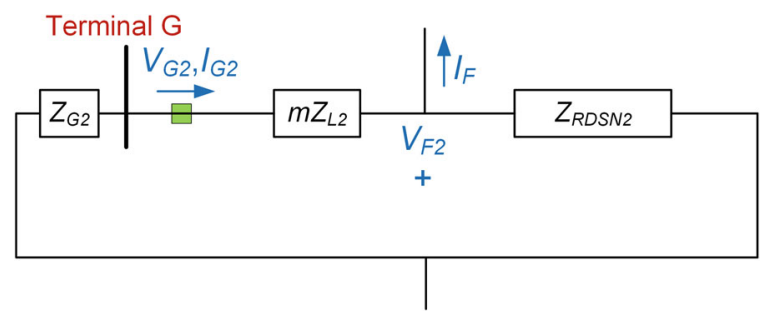

Fig. 8 Negative-sequence network of a general transmission line network during an unbalanced fault

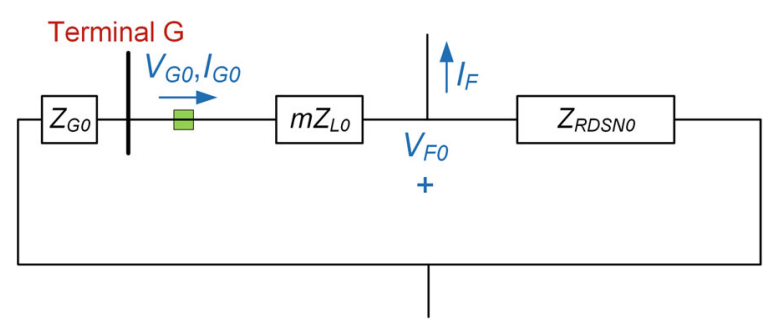

Fig. 9 Zero-sequence network of a general transmission line network during a ground fault

ponents such as lines and transformers. However, the sequence impedances are not equal to each other in rotating machines.

\section{Demonstration of estimation of Thevenin impedance of upstream network using a test case}

To demonstrate the approach described in Section 4 for estimating the Thevenin impedance of the upstream network, the three-terminal line test case analyzed in Section 3 is used. The same fault scenario, a single lineto-ground (SLG) fault on phase A at a distance of 13 miles from terminal $\mathrm{G}$ with monitors at terminal $\mathrm{G}, \mathrm{H}$ and $\mathrm{T}$ is used. The current and voltage waveforms recorded at terminal $\mathrm{G}$ are shown in Fig. 11.
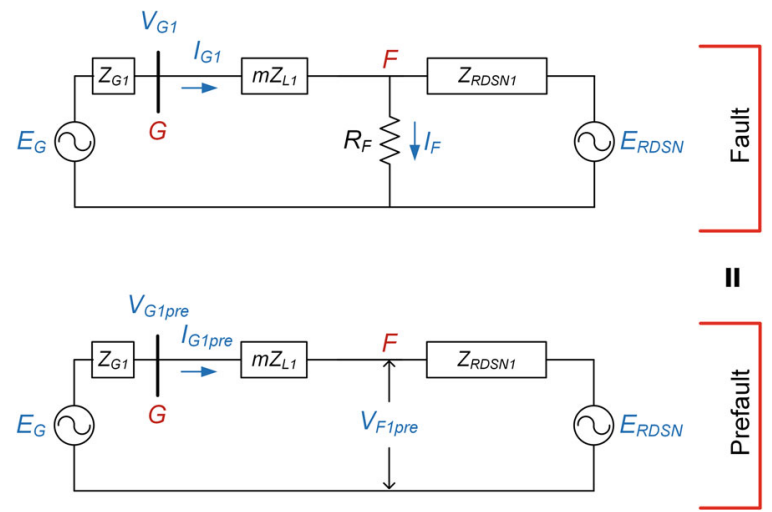

II
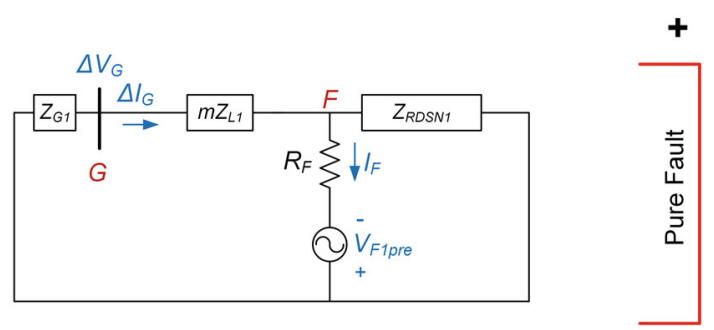

Fig. 10 Superposition theorem used to decompose the network into a prefault and a "pure" fault during a three-phase fault 

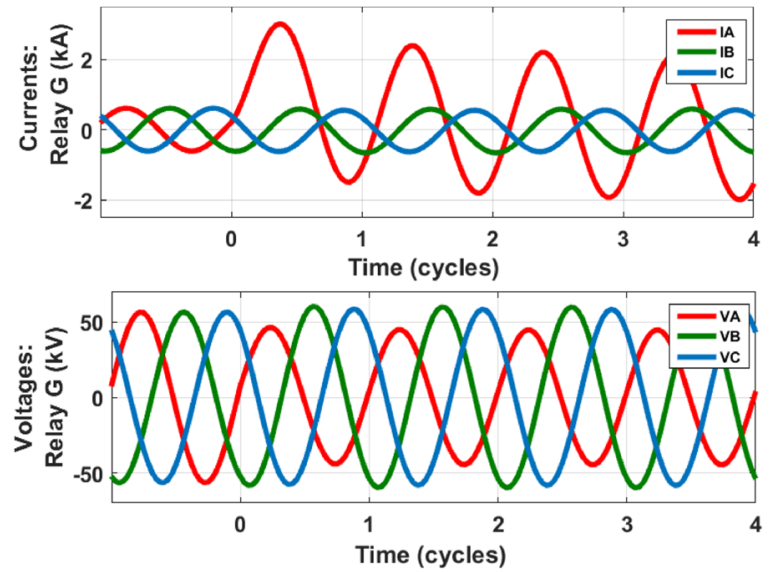

Fig. 11 Current and voltage waveforms recorded at terminal G. IA, IB and $I C$ are the currents recorded in phases $A, B$ and $C$ respectively at terminal G. VA, VB and $V C$ are the phase-ground voltages recorded in phases $A, B$ and $C$ respectively at terminal $G$

From the waveforms recorded by the relay at terminal $\mathrm{G}$, the sequence voltage and current phasors before and during the fault are:

$$
\begin{array}{ll}
V_{G 1}=38.35 \angle 63.28^{\circ} \mathrm{kV} & I_{G 1}=0.64 \angle 30.36^{\circ} \mathrm{kA} \\
V_{G 2}=1.67 \angle-106.16^{\circ} \mathrm{kV} & I_{G 2}=0.45 \angle-12.16^{\circ} \mathrm{kA} \\
V_{G 0}=5.18 \angle-105.67^{\circ} \mathrm{kV} & I_{G 0}=0.46 \angle-11.67^{\circ} \mathrm{kA} \\
V_{G 1 \text { pre }}=40 \angle 63.86^{\circ} \mathrm{kV} & I_{G 1 \text { pre }}=0.43 \angle 75.13^{\circ} \mathrm{kA}
\end{array}
$$

The above phasors are then used in (17) - (19) to calculate the Thevenin impedance of the upstream network of Terminal G. Table 3 presents the estimated Thevenin impedances at Terminal G.

As seen from Table 3, the estimated source impedances matched well with the actual impedances used in the simulation model. The magnitude error percentages of the estimated Thevenin impedances are less than $1 \%$. The positive-sequence impedance shows a small deviation from the actual value. Most likely, the error stems from the constant current load model assumption in (19). Similarly, the Thevenin impedance can be estimated at Terminals $\mathrm{H}$ and $\mathrm{T}$ as well using sequence currents measured at their respective terminals.

Table 3 Actual vs. Estimated Thevenin impedances at Terminal $\mathrm{G}$ for the three-terminal line test scenario

\begin{tabular}{lllll}
\hline & \multicolumn{2}{l}{$\begin{array}{l}\text { Source } \\
\text { impedance }(\Omega)\end{array}$} & $\begin{array}{l}\text { Magnitude } \\
\text { error (\%) }\end{array}$ & $\begin{array}{l}\text { Phase angle } \\
\text { error (degrees) }\end{array}$ \\
\cline { 2 - 3 } & Actual & Estimated & & \\
\hline$Z_{G 1}$ & $3.75 \angle 86^{\circ}$ & $3.78 \angle 89.2^{\circ}$ & 0.80 & 3.2 \\
$Z_{G 2}$ & $3.75 \angle 86^{\circ}$ & $3.75 \angle 86^{\circ}$ & 0.00 & 0.00 \\
$Z_{G 0}$ & $11.25 \angle 86^{\circ}$ & $11.25 \angle 86^{\circ}$ & 0.00 & 0.00 \\
\hline
\end{tabular}

The process of estimating the Thevenin impedance of the upstream network is done for each terminal of a transmission line. It requires data from only one terminal to estimate the Thevenin impedance of the upstream network of that terminal as shown in (17) - (19). It does not require data or information from other terminals of the line.

The calculation of Thevenin impedance or source impedance is done for the upstream network. Hence, the upstream Thevenin impedance is not affected by components downstream of the monitoring location. Therefore, the transmission line that is present after the monitor does not affect the Thevenin impedance of the upstream network.

\section{Conclusion}

This paper presents methods to estimate the zerosequence line impedance of all the sections in a threeterminal transmission line using fault data from all three terminals as well as using data from only two terminals. When data from only two terminals are available, the algorithm requires source impedance data from the third terminal, assumes that the fault location is known and the fault resistance is zero. These assumptions are not required when data from all three terminals are available. The accuracy of the proposed methods are demonstrated using a test case. Both approaches produced accurate zero-sequence line impedance estimates when the assumptions were satisfied and the magnitude error percentage was less than $1 \%$. Additionally, the paper also presented a technique for estimating Thevenin equivalent impedance of the transmission network upstream from the monitoring location. The efficacy of this method was demonstrated using a test case. The magnitude error percentage in estimating the Thevenin impedance was less than $1 \%$ in the test case. Hence, it can be concluded that the presented methods provide a reliable estimation of the zero-sequence impedance of the line and Thevenin impedance of the upstream network which can be used to verify relay settings.

\section{Authors' contributions}

SD and SNA had developed the algorithms, performed the analysis and drafted the manuscript. SS was the technical advisor and supervised the analysis and submission of the manuscript. All authors read and approved the manuscript.

Competing interests

The authors declare that they have no competing interests.

\section{Author details}

${ }^{1}$ Schweitzer Engineering Laboratories, Pullman, USA. ${ }^{2}$ Department of Electrical and Computer Engineering, The University of Texas, Austin, USA.

Received: 4 March 2018 Accepted: 26 September 2018

Published online: 06 November 2018 


\section{References}

1. Costello, D. (2008). Lessons learned analyzing transmission faults, In 2008 61st Annual Conference for Protective Relay Engineers, College Station, TX (pp. 410-422).

2. Das, S., \& Santoso, S. (2016). Utilizing relay event reports to identify settings error and avoid relay misoperations, In 2016 IEEE/PES Transmission and Distribution Conference and Exposition (T\&D), Dallas, TX (pp. 1-5).

3. Das, S., Navalpakkam Ananthan, S., Santoso, S. (2018). Relay performance verification using fault event records. Protection and Control of Modern Power Systems, 3(1), 22.

4. Ritzmann, D., Wright, P.S., Holderbaum, W., Potter, B. (2016). A method for accurate transmission line impedance parameter estimation. IEEE Transactions on Instrumentation and Measurement, 65(10), 2204-2213.

5. Das, S., Ananthan, S.N., Santoso, S. (2017). Estimating zero-sequence line impedance and fault resistance using relay data. IEEE Transactions on Smart Grid, PP(99), 1-1.

6. Amberg, A., Rangel, A., Smelich, G. (2012). Validating transmission line impedances using known event data, In 2012 65th Annual Conference for Protective Relay Engineers, College Station, TX (pp. 269-280).

7. Zimmerman, K, \& Costello, D (2010). Fundamentals and improvements for directional relays, In 2010 63rd Annual Conference for Protective Relay Engineers, College Station, TX (pp. 1-12).

8. Das, S., Santoso, S., Gaikwad, A., Patel, M. (2014). Impedance-based fault location in transmission networks: theory and application. IEEE Access, 2, 537-557.

9. Sundaravaradan, N.A, \& Reddy, M.J.B. (2018). How is earthing done? IEEE Potentials, 37(2), 42-46.

10. (2006). The complexity of protecting three-terminal transmission lines, System Protection and Control Task Force of the NERC Planning Commitee. Available: http://www.nerc.com/comm/PC/System \%20Protection\%20and\%20Control\%20Subcommittee\%20SPCS\%20DL/ SPCTF-3TerminalLinesX091906.pdf. Accessed 4 Mar 2018.

11. Schweitzer III, E.O. (1990). A review of impedance-based fault locating experience, In 14th Annual lowa-Nebraska System Protectection Seminar.

12. Santoso, S. (2009). Fundamentals of Electric Power Quality, Spring ed. Scotts Valley: CreateSpace.

13. Eriksson, L., Saha, M.M., Rockefeller, G.D. (1985). An accurate fault locator with compensation for apparent reactance in the fault resistance resulting from remote-end infeed. IEEE Transactions on Power Apparatus and Systems, PAS-104(2), 423-436.

14. Novosel, D., Hart, D., Hu, Y., Myllymaki, J. (1998). System for locating faults and estimating fault resistance in distribution networks with tapped loads. U.S. Patent 5839093.

15. Sharma, C., \& Castellanos, F. (2006). Remote fault estimation and Thevenin impedance calculation from relays event reports, In 2006 IEEE/PES Transmission \& Distribution Conference and Exposition: Latin America, Caracas (pp. 1-7).

16. Sun, Y., Dai, C., Li, J. (2015). Thevenin equivalent circuit parameter estimation for the PCC upstream system, In 2015 5th International Conference on Electric Utility Deregulation and Restructuring and Power Technologies (DRPT), Changsha (pp. 2299-2303).

17. PSCAD User's Guide [Online] Available: https://hvdc.ca/uploads/ knowledge_base/pscad_user_guide_v4_3_1.pdf?t=1462310124. Accessed 24 Sept 2018.

18. Kersting, W.H. (2012). Distribution System Modeling and Analysis, 3rd ed. Boca Raton: CRC Press.

19. Sundaram, A. (2006). Distribution fault location: field data and analysis. Palo Alto: EPRI. (1012438).

20. Min, K.W., \& Santoso, S. (2017). DC offset removal algorithm for improving location estimates of momentary faults. IEEE Transactions on Smart Grid, PP(99), 1-1.

21. Zamora, A., Ramirez, J.M., Paternina, M.R.A., Vazquez-Martinez, E. (2015). Digital filter for phasor estimation applied to distance relays. IET General Transactions Distribution, 9(14), 1954-1963.

\section{Submit your manuscript to a SpringerOpen ${ }^{\circ}$ journal and benefit from:}

\section{- Convenient online submission}

- Rigorous peer review

- Open access: articles freely available online

- High visibility within the field

- Retaining the copyright to your article

Submit your next manuscript at $\gg$ springeropen.com 